\title{
Validación de un puntaje de riesgo cardiovascular en niños españoles aplicado a una población de escolares de Santiago de Chile
}

\author{
PILAR ARNAIZ ${ }^{1,2}$, FELIPE PINO $^{\mathrm{a}}$, ARNALDO MARÍN ${ }^{\mathrm{a}}$, \\ SALESA BARJA $^{1}$, MARLENE AGLONY ${ }^{1}$, BERTA CASSIS ${ }^{1}$, \\ CARLOS NAVARRETE ${ }^{3 b}$, MÓNICA ACEVEDO ${ }^{2}$
}

'División de Pediatría. ${ }^{2}$ División de Enfermedades Cardiovasculares

Pontificia Universidad Católica de Chile, Santiago, Chile. ${ }^{3}$ Departamento de Matemática, Universidad de la Serena, La Serena, Chile. aAlumno Medicina, Facultad de Medicina, Pontificia Universidad Católica de Chile. ${ }^{b}$ Doctor en Estadística.

Recibido el 21 de enero de 2010, aceptado el 15 de septiembre de 2010

Correspondencia a: Dra. Mónica Acevedo División de Enfermedades Cardiovasculares, Lira 85, Primer Piso, Santiago, Chile

Teléfono: 56-2-3543334 E-mail: macevedo@med.puc.cl

\section{Association between a cardiovascular risk score and early markers of atherosclerotic disease in Chilean children}

\begin{abstract}
Background: A cardiovascular risk score for children, that includes traditional risk factors, obesity, sedentary habits and a family history of cardiovascular disease, has been recently proposed by Spanish researchers. Aim: To apply this score in school age children in Santiago de Chile and correlate its results with markers of subclinical atherosclerotic disease. Material and Methods: Retrospective analysis of data obtained from 209 children, aged $11.5 \pm 2$ years, studied between 2005 and 2006. Weight, height, blood pressure, ultrasound measurement of carotid intima-media thickness and flow mediated dilatation of brachial artery and ultrasensible $C$ reactive protein (us PCR) were measured. The Spanish cardiovascular risk score was calculated and correlated with ultrasound parameters and C reactive protein. Results: According to the score, 173 children (83\%) had a low cardiovascular risk, 28 (13\%) an intermediate risk and $8(4 \%)$ a high risk. There was no association between the cardiovascular risk score and carotid intima-media thickness, flow mediated arterial dilatation and us PCR. Conclusions: No significant association was observed between the proposed cardiovascular risk score and early markers of atherosclerotic disease in this group of children.

(Rev Med Chile 2010; 138: 1226-1231).
\end{abstract}

Key words: Atherosclerosis; Cardiovascular diseases; Childhood.
L a epidemia de obesidad declarada por la Organización Mundial para la Salud (OMS) ha alcanzado niveles alarmantes en niños y jóvenes de Chile, con 18,5\% de prevalencia en los escolares de primero básico, según estadísticas de la Junta Nacional de Auxilio Escolar y Becas (JUNAEB $)^{1}$. En los niños, tanto la obesidad general como la adiposidad visceral, se asocian a mayor riesgo cardiovascular y metabólico en la vida adulta, independiente del peso que se presente en aquella edad ${ }^{2}$. Esto se compadece con la evidencia científica que ha demostrado el inicio precoz del proceso ateroesclerótico en niños obesos con factores de riesgo cardiovascular ${ }^{3}$. Si bien esto ha sido probado mediante estudios anatomopatológicos en arterias de niños en los que se ha demostrado lesiones ateroscleróticas tempranas, actualmente se ha logrado trabajar con marcadores estructurales, funcionales y bioquímicos ${ }^{4,5}$ de enfermedad ateroesclerótica subclínica, los cuales pueden ser evaluados antes que los puntos finales duros.

La proteína C-reactiva ultrasensible (PCRus), es un marcador bioquímico de aterosclerosis. Es la principal proteína de fase aguda inflamatoria y 
se asocia, tanto estadística como fisiopatológicamente, con la enfermedad ateroesclerótica y con sus factores de riesgo, aun en edades tempranas. De esta forma, la PCRus se ha encontrado en lesiones ateroescleróticas en piezas de anatomía patológica, y se ha demostrado de forma directa la inducción de células inflamatorias y moléculas de adhesión en la íntima, además de disminuir la óxido nítrico sintetasa $(\mathrm{NOS})^{6}$.

Por otro lado, la ultrasonografía de alta resolución ha resultado ser una excelente herramienta en la pesquisa temprana de la pérdida de la dilatación dependiente del endotelio y del engrosamiento arterial, siendo conocidas herramientas en la actualidad como fenotipos o marcadores intermedios de la enfermedad ateroesclerótica. Es así como se ha demostrado la asociación entre valores altos de grosor de íntima media carotídea (IMT) y cifras bajas de dilatación mediada por flujo de la arteria braquial (DMF) en niños obesos con factores cardiovasculares y metabólicos ${ }^{7,8}$.

El gran problema en la evaluación del riesgo cardiovascular en niños es que, a diferencia de los adultos, en que tenemos puntajes de valoración basados en puntos finales duros o llamados eventos (ej., puntaje de Framingham), en niños no podemos esperar puntos finales. En este contexto es que se propuso como objetivo de este estudio, el aplicar un puntaje de riesgo cardiovascular (RCV) a una población de escolares de Santiago, buscando asociación con puntos intermedios, a través del uso de marcadores estructurales, funcionales y bioquímicos de ateroesclerosis subclínica. De esta manera se buscó validar un puntaje de advertencia que fuera aplicable, fácilmente, en el escenario ambulatorio, permitiendo establecer intervenciones efectivas y controladas, ya que es en este período cuando las medidas preventivas podrían tener su mayor efecto?.

\section{Métodos}

La aplicación de la encuesta se realizó a una población ya estudiada por el mismo grupo y que se describe y explicitan los exámenes efectuados a continuación.

Se estudiaron retrospectivamente los datos de 209 escolares entre 6 y 16 años, de estrato socioeconómico medio, medio bajo y bajo de la Región Metropolitana de Santiago, estudiados en los años 2005 y 2006. En estos niños se registraron datos sobre antecedentes familiares y personales de RCV. Paralelamente se utilizó la evaluación clínica (peso, talla y presión arterial), de laboratorio (perfil lipídico y PCRus) y ultrasonográfica (IMT y DMF). Finalmente, se aplicó el puntaje de RCV propuesto por Alústiza y $\mathrm{cols}^{10}$. Todo este proceso se realizó con previa autorización del adulto responsable, con firma de consentimiento informado para participación en el estudio inicial, aprobado por el Comité de Ética de la Pontificia Universidad Católica de Chile.

Evaluación antropométrica y clínica: En cada niño se midió peso y talla en forma controlada, con balanza de palanca y estatímetro marca SECA ${ }^{\circledR}$, descalzos, vestidos con ropa interior y una bata. Se calculó el índice de masa corporal (IMC: Peso (kg)/ Talla $^{2}(\mathrm{~m})$ ), expresado en percentiles. Se definió obesidad como índice de masa corporal (IMC) igual o mayor al percentil 95 para edad y sexo y eutrofia entre percentil 10 y 84 . Se utilizaron como referencia las curvas NCHS-CDC año $2000^{11}$ y las normas para evaluación nutricional del niño del Ministerio de Salud ${ }^{12}$.

La presión arterial se tomó en tres controles cada 2 minutos, en decúbito supino, después de 10 minutos de reposo y en el brazo derecho, promediándose los valores. Se utilizó un equipo oscilométrico automático (Dynamap Pro 100, Criticon ${ }^{\circledR}$ ) con manguito pediátrico escolar y adulto. Se consideró hipertensión si la presión sistólica o diastólica era mayor o igual al p95 para sexo, edad y talla ${ }^{13}$.

Evaluación sérica: La PCRus fue medida por método nefelométrico Dade Behring (Nefelómetro Dade Behring BN II) con límite de detección de $0,1 \mathrm{mg} / \mathrm{L}$. El colesterol total, CHDL y triglicéridos fueron medidos usando los métodos enzimáticos estándar. Se calculó CLDL mediante la fórmula de Friedwald (LDL $=$ CT- $($ TG $/ 5+\mathrm{HDL})$ ).

Evaluación ultrasonográfica: Los exámenes ultrasonográficos y la lectura de ellos fueron efectuados por dos ecografistas entrenadas y certificadas.

\section{Estudio de función endotelial}

Se realizó según las recomendaciones internacionales ${ }^{14}$. Se utilizó un equipo Hewlett Packard Sonos 5500 con un transductor lineal de alta frecuencia (5-13 Mhz). Con el niño en posición supina y confortable, pieza con temperatura controlada, se colocó un manguito en el antebrazo 
no dominante. Se escaneó la arteria braquial en forma horizontal por sobre el pliegue del codo, para obtener una visión óptima de las interfases lumen-íntima para hacer la medición basal. Se procedió con la fase de hiperemia, la que se indujo por presión aplicada sobre el antebrazo con un manguito que se infló al menos $50 \mathrm{~mm} \mathrm{Hg}$ por sobre la presión sistólica de reposo, por 5 minutos. Al liberarse el manguito, se produjo la respuesta hiperémica. La medición del diámetro de la arteria post hiperemia reactiva se efectuó entre los 45 y 60 segundos post liberación del manguito, en fin de diástole, en 3 ciclos cardíacos. Se midió de íntima a íntima, utilizando un cáliper electrónico (software M’Ath $^{\circledR}$ Std). El porcentaje de dilatación de la arteria se calculó al restar el diámetro de la arteria post hiperemia menos el diámetro basal, dividido por el diámetro basal multiplicado por 100 . No se realizó la etapa de dilatación independiente del endotelio con nitroglicerina por la edad de los niños y el peligro de hipotensión.

\section{Grosor Intima-Media Carotídeo (IMT)}

Se realizó según las recomendaciones del consenso en medición del grosor mio-intimal carotídeo ${ }^{15}$. Para la medición del IMT se utilizó el mismo transductor y equipo ecográfico detallado previamente. La imagen se focalizó en la pared posterior de cada arteria carótida común, donde se eligió un segmento de $1 \mathrm{~cm}$, proximal a la bifurcación carotídea de cada lado. Para la medición se usó un software con detección automática de bordes (M’Ath ${ }^{\circledR}$ Std). Todas las mediciones se realizaron "off-line" y en fin de diástole. El valor de IMT medio y máximo registrados para el análisis estadístico correspondió al valor de IMT entre el lado derecho e izquierdo.

Puntaje de RCV: Se aplicó un puntaje modificado (Tabla 1) a partir del propuesto por Alústiza y cols ${ }^{10}$ en España, que considera edad, sexo, antecedentes familiares, actividad física, tabaquismo, obesidad general, presión arterial y colesterol LDL. En este estudio consideramos los antecedentes familiares bioquímicos positivos, si uno o ambos padres tenían antecedente positivo de dislipidemia (DLP). Por otro lado, los antecedentes familiares clínicos positivos considerados para uno o ambos padres fueron la cardiopatía coronaria o accidente

Tabla 1. Puntaje de RCV aplicado a I a muestra en estudio

\begin{tabular}{|lll|}
\hline Variable & Valor & Puntuación \\
\hline \multirow{2}{*}{ Edad } & 2 a 5 años & 0 puntos \\
& 6 a 12 años & 2 puntos \\
& $>13$ años & 3 puntos \\
Antecedentes familiares & Mujer & 0 puntos \\
& Varón & 2 puntos \\
Ejercicio & Ausentes & 0 puntos \\
& (+) Bioquímicos & 2 puntos \\
Tabaco/alcohol & (+) Clínicos & 4 puntos \\
Obesidad (IMC) & $>2$ hr/día y TV $<3$ hr/día & 0 puntos \\
& $<2$ hr/día y TV $>3$ hr/día & 1 punto \\
Presión arterial & No & 0 puntos \\
& Sí & 1 punto \\
Colesterol & $<$ Percentil 95 & 0 puntos \\
& $>$ Percentil 95 & 1 punto \\
& $<$ Percentil 95 & 0 puntos \\
& $>$ Percentil 95 & 1 punto \\
& CT: 150-199, LDL 100-109 & 0 punto \\
& CT: 200-220, LDL 110-130 & 1 punto \\
& CT. 221-230, LDL 131-160 & 2 puntos \\
& CT: 231-280, LDL 161-190 & 3 puntos \\
& CT $>281$, LDL $>190$ & 6 puntos \\
\hline
\end{tabular}


Tabla 2. Marcadores de ATE subclínica para cada estrato de puntaje de riesgo cardiovascular

\begin{tabular}{|lcccc|}
\hline & $\begin{array}{c}\text { Puntaje Bajo } \\
\text { (0 a 6 puntos) }\end{array}$ & $\begin{array}{c}\text { Puntaje Medio } \\
\text { (7-8 puntos) }\end{array}$ & $\begin{array}{c}\text { Puntaje Alto } \\
\text { (9 o más puntos) }\end{array}$ & $\begin{array}{c}\text { Valor "p" } \\
\text { (tendencia lineal) }\end{array}$ \\
\hline IMT medio (mm) & $0,49 \pm 0,03$ & $0,50 \pm 0,03$ & $0,53 \pm 0,05$ & 0,09 \\
\hline IMT máximo (mm) & $0,60 \pm 0,06$ & $0,59 \pm 0,09$ & $0,63 \pm 0,10$ & 0,8 \\
\hline DMF (\%) & $9,36 \pm 4,3$ & $9,11 \pm 3,3$ & $8,62 \pm 1,4$ & 0,75 \\
PCRus (mg/dl) & $1,24(2,3)$ & $1,37(1,2)$ & $2,44(1,8)$ & 0,052 \\
\hline
\end{tabular}

IMT: Grosor íntima-media carotídea; DMF: Dilatación mediada por flujo de la arteria braquial; PCRus: PCR ultrasensible.

vascular encefálico prematuro (antes de los 55 años en varones, y antes de 65 años en mujeres).

El Puntaje de Alustiza y cols ${ }^{10}$ oscila desde 0 a 21 puntos como máximo, logrando categorizar la muestra en tres niveles de puntajes (bajo: 0 a 6 puntos; medio: 7-8 puntos; alto: 9 o más puntos), que demostrarían probabilidad creciente de desarrollar enfermedad ateroesclerótica subclínica.

\section{Análisis Estadístico}

Se ajustaron modelos de regresión logística para estudiar la asociación entre el puntaje (bajo, moderado y alto), y la probabilidad de presentar niveles elevados de IMT, PCRus, como también niveles bajos de DMF. Se consideró nivel elevado el percentil 75 y bajo el percentil 25 de la misma muestra de niños. Se compararon los niveles de IMT, DMF y logPCR en escala continua mediante ANOVA unifactorial. Se consideró significancia estadística para $\mathrm{p}<0,05$. Los resultados fueron obtenidos del software estadístico R.

\section{Resultados}

La edad promedio de la muestra fue de 11,5 \pm 2 años, siendo $50 \%$ mujeres y $30 \%$ prepúberes. De acuerdo al IMC, 53\% de los niños eran eutróficos, $18 \%$ sobrepeso y $29 \%$ obesos. En base al puntaje aplicado, 173 niños (83\%) clasificaron para puntaje bajo (0-6 puntos), 28 (13\%) para medio (7-8 puntos), y sólo 8 niños (4\%) para puntaje alto ( 9 o más puntos).

Respecto al puntaje y los marcadores de ateroesclerosis subclínica (Tabla 2) observamos tendencias crecientes en los estimadores puntuales para la media de IMT y PCRus, y una tendencia decreciente para DMF. Esta tendencia, sin embargo, no fue significativa desde el punto de vista estadístico. Al aplicar el puntaje como predictor de aumento de riesgo de presentar valores elevados de IMT y PCRus (ambos con un punto de corte determinado por el percentil 75 de la muestra), el efecto no resultó estadísticamente significativo en términos de odds ratio. El mismo análisis para valores bajos de DMF (menor al percentil 25) tampoco se tradujo en diferencias significativas.

En base a estos resultados, se concluye que, en este estudio, no se encontró asociación significativa entre el puntaje de RCV aplicado y los marcadores de ATE subclínica medidos.

\section{Discusión}

La obesidad en la niñez y adolescencia constituye un fenómeno creciente ${ }^{16}$, la cual se mantiene presente en la vida adulta en $77 \%$ de los casos, según lo reportado por los estudios Muscatine ${ }^{17} \mathrm{y}$ The Cardiovascular Risk in Young Finns ${ }^{18}$. Por otro lado, de forma semejante a los adultos, la obesidad infantil se asocia a otros factores de RCV, como hipertensión arterial, dislipidemia y alteraciones del metabolismo de la glucosa, cuya presencia conjunta constituye el llamado síndrome metabólico o de resistencia insulínica ${ }^{19,20}$, determinando mayor riesgo cardiovascular y morbimortalidad, que ha sido evidenciado con la presencia de ateroesclerosis temprana en anatomía patológica ${ }^{3}$.

Si bien nuestro estudio no arrojó resultados estadísticamente significativos, se logra evidenciar una tendencia entre los marcadores de ateroesclerosis subclínica y el puntaje de RCV, como son la tendencia directa con IMT y PCRus, e inversa con DMF. Estos resultados nos hacen plantear que una de las principales limitaciones del estudio es la falta de potencia estadística debido al tamaño muestral de 209 niños. Por otro lado, nuestros hallazgos 
pueden ejemplificar lo difícil de la medición de estos fenotipos intermedios en la población pediátrica, pues es sabido que los factores de RCV deben cumplir un tiempo mínimo de exposición y cierto tiempo de agregación, para poder potenciarse y manifestarse a niveles anátomo y fisiopatológicos ${ }^{21}$. En niños, este tiempo de exposición y agregación es muy difícil de determinar. Si a esto le sumamos el hecho de estar en presencia de un estudio transversal, parece razonable pensar que, al momento de las mediciones, pudieron no haber existido diferencias que permitieran lograr la significancia estadística entre las variables estudiadas. Por último, en esta investigación, nosotros consideramos alterado un valor de IMT y PCRus $>$ percentil 75, y para DMF $<$ a percentil 25 de esta misma muestra, sabiendo que estos cortes pueden ser arbitrarios, ya que están definidos como patológicos en adultos, pero no en niños. Sin embargo, este estudio es pionero en la literatura nacional e internacional en cuanto a la utilización de marcadores intermedios de aterosclerosis en niños y su relación con factores de riesgo cardiovascular ponderados a través de un puntaje de riesgo.

En base a lo expuesto, se plantea que para la construcción de un puntaje de RCV se deben realizar estudios prospectivos, con muestras grandes y representativas en donde se midan estos biomarcadores intermedios. De esta forma, se podrán realizar modelos estadísticos que expliquen las tendencias que se puedan observar para, posteriormente, crear el puntaje. Así se podría garantizar reproducibilidad.

La importancia de la realización de instrumentos para categorizar el riesgo cardiovascular en la edad pediátrica (como el que se propone en este estudio) radica en la dificultad existente en la actualidad para intervenir en forma más agresiva esta población. En este sentido, en adultos, se ha demostrado que la utilización de marcadores intermedios, como el grosor de la carótida, ha permitido una mejor adherencia de los pacientes a sus tratamientos ${ }^{22}$. Si la agregación de factores de riesgo cardiovascular en la edad pediátrica se asociara con la presencia de marcadores intermedios, esto permitiría categorizar mejor a aquellos niños y adolescentes que requirieran de una intervención más precoz y agresiva.

Obviamente, nuestro estudio tiene limitaciones, que se han ya detallado: es un estudio en que el puntaje se aplicó en forma retrospectiva.
El número de niños de la muestra es pequeño, pero estos niños estaban muy bien estudiados, $y$ no hubo carencia de datos para llenar la encuesta.

En resumen, conocida la problemática del sobrepeso y obesidad en los niños y su demostrada asociación a mayor riesgo cardiovascular y metabólico en la vida adulta, es relevante tener un rol activo en el reconocimiento de la enfermedad ateroesclerótica subclínica, para poder realizar intervenciones más precoces y efectivas. La creación de un puntaje que traduzca advertencia de ateroesclerosis en niños nos parece una herramienta costo-efectiva para niveles ambulatorios. En nuestro estudio se evidenciaron las tendencias esperables respecto al nivel de puntaje y los marcadores de enfermedad subclínica (IMT, DMF y PCRus). La significancia estadística y reproducibilidad se podrían garantizar al realizar estudios prospectivos en muestras grandes y representativas.

\section{Referencias}

1. Estadísticas junaeb. www.junaeb.cl. 2005.

2. Must A, Jacques PF, Dallal GE, Bajema CJ, Dietz WH. Long-term morbidity and mortality of overweight adolescents. A follow-up of the Harvard Growth Study of 1922 to 1935. N Engl J Med 1992; 327: 1350-5.

3. Berenson GS, Srinivasan SR, Bao W, Newman WP, 3rd, Tracy RE, Wattigney WA. Association between multiple cardiovascular risk factors and atherosclerosis in children and young adults. The Bogalusa Heart Study. N Engl J Med 1998; 338: 1650-6.

4. Barja S, Acevedo M, Arnaiz P, Berríos X, Bambs C, Guzmán $\mathrm{B}$, et al. Marcadores de aterosclerosis temprana y síndrome metabólico en niños. Rev Med Chile 2009; 137: 522-30.

5. Acevedo M, Arnaiz P, Barja S, Berríos X, Bambs C, Guzmán $\mathrm{B}$, et al. Proteína c-reactiva y su relación con adiposidad, factores de riesgo cardiovascular y ateroesclerosis subclínica en niños sanos de la Región Metropolitana. Revista Chilena de Cardiología 2007; 26: 43-54.

6. Lagrand WK, Visser CA, Hermens WT, Niessen HW, Verheugt FW, Wolbink GJ, et al. C-reactive protein as a cardiovascular risk factor: More than an epiphenomenon? Circulation 1999; 100: 96-102.

7. Beauloye V, Zech F, Tran HT, Clapuyt P, Maes M, Brichard SM. Determinants of early atherosclerosis in obese children and adolescents. J Clin Endocrinol Metab 2007; 92: 3025-32.

8. Pena AS, Wiltshire E, MacKenzie K, Gent R, Piotto L, Hirte $\mathrm{C}$, et al. Vascular endothelial and smooth muscle 
function relates to body mass index and glucose in obese and nonobese children. J Clin Endocrinol Metab 2006; 91: 4467-71.

9. Celermajer DS. Primary and a half prevention: Can we identify asymptomatic subjects with high vascular risk? J Am Coll Cardiol 2005; 45: 1994-6.

10. Alústiza E, Blarduni E, Aldámiz-Echeverría L, Aranzábal M, Ugarte R, Gorostiza E, et al, Grupo KURSAAL. Presentación de un score en la prevención de la enfermedad cardiovascular. An Esp Pediatr 1997; 108: 63-5.

11. National Health and Nutrition Examination Survey. Anthropometry procedures manual. Http//www.Cdc. Gov/nchs/data/nhanes/bm.Pdf 2002. 2002.

12. Minsal. Unidad de nutrición: Norma para la evaluación nutricional del niño entre 6 y 18 años. 2003.

13. Update on the task force report on high blood pressure in children and adolescents: A working group report from the national high blood pressure education program, national institute of health. NIH publication. 1996; 96: 3790 .

14. Corretti MC, Anderson TJ, Benjamin EJ, Celermajer D, Charbonneau F, Creager MA, et al. Guidelines for the ultrasound assessment of endothelial-dependent flowmediated vasodilation of the brachial artery: A report of the international brachial artery reactivity task force. J Am Coll Cardiol 2002; 39: 257-65.

15. Touboul PJ, Hennerici MG, Meairs S, Adams H, Amarenco P, Desvarieux M, et al. Mannheim intima-media thickness consensus. Cerebrovasc Dis 2004; 18: 346-9.
16. Ebbeling CB, Pawlak DB, Ludwig DS. Childhood obesity: Public-health crisis, common sense cure. Lancet 2002; 360: 473-82.

17. Davis PH, Dawson JD, Riley WA, Lauer RM. Carotid intimal-medial thickness is related to cardiovascular risk factors measured from childhood through middle age: The Muscatine study. Circulation 2001; 104: 2815-9.

18. Raitakari OT, Juonala M, Kahonen M, Taittonen L, Laitinen T, Maki-Torkko N, et al. Cardiovascular risk factors in childhood and carotid artery intima-media thickness in adulthood: The Cardiovascular Risk in Young Finns study. JAMA 2003; 290: 2277-83.

19. Barja S, Arteaga A, Acosta AM, Hodgson MI. Resistencia insulínica y otras expresiones del síndrome metabólico en niños obesos chilenos. Rev Med Chile 2003; 131: 25968.

20. Cook S, Weitzman M, Auinger P, Nguyen M, Dietz WH. Prevalence of a metabolic syndrome phenotype in adolescents: Findings from the third national health and nutrition examination survey, 1988-1994. Arch Pediatr Adolesc Med 2003; 157: 821-7.

21. Tounian P, Aggoun Y, Dubern B, Varille V, Guy-Grand $B$, Sidi D, et al. Presence of increased stiffness of the common carotid artery and endothelial dysfunction in severely obese children: A prospective study. Lancet 2001; 358: 1400-4.

22. Sharma K, Blaha MJ, Blumenthal RS, Musunuru K. Clinical and research applications of carotid intima-media thickness. Am J Cardiol 2009;103: 1316-20. 